\title{
Method of DTM Extraction and Visualization using Threshold Segmentation and Mathematical Morphology
}

\author{
Tianyong $\mathrm{Wu}^{\mathrm{a}}$, Yunsheng Zhao ${ }^{\mathrm{a}, *}$, and Xiang $\mathrm{Li}^{\mathrm{b}}$ \\ ${ }^{a}$ Faculty of Engineering, China University of Geosciences, Wuhan, 430074, China \\ ${ }^{b}$ School of Computer Science, China University of Geosciences, Wuhan, 430074, China
}

\begin{abstract}
LiDAR (Light Detection and Ranging) is a laser ranging technology that provides an efficient and convenient way to obtain the original data from DSM (Demand Side Management). The basic task of LiDAR is to separate the high quality DTM (Digital Terrain Model) from the DSM, and the accuracy and quality of the generated image are determined by the different filtering and interpolation algorithms. According to this, this paper presents a filtering algorithm based on the optimal threshold segmenting optimized by the erosion operation (OTS-EO) to improve the problem that the manually-set-height difference threshold is empirically affected. In order to overcome the deficiency of the distance-based IDP (Inverse Distance to a Power) interpolation algorithm, an interpolation algorithm based on elevation and distance weighting is proposed to obtain the DSM to be further filtered. In this paper, the original laser point cloud data near the Xinyan rode in Beijing is taken as an example, and the data is processed by the algorithm based on threshold segmentation and mathematical morphology (TSMM) to extract the DTM. Finally, the 3D visualization of DTM is realized by the program based on MFC and OpenGL. The experimental data and practices in engineering show that the TSMM algorithm can successfully separate and display the surface points and surface features and extract the DTM close to the real ground to provide the foundation for further research.
\end{abstract}

Keywords: LiDAR; threshold segmentation; mathematical morphology; digital terrain model; 3D visualization

(Submitted on October 25, 2018; Revised on November 23, 2018; Accepted on December 26, 2018)

(C) 2019 Totem Publisher, Inc. All rights reserved.

\section{Introduction}

The application of LiDAR point cloud data has a great potential in the establishment of DTM. Since the original LiDAR point cloud data is discrete and irregularly distributed, the original data needs to be processed subsequently to extract the accurate and meaningful DTM. At present, many scholars have made corresponding research and contributions on the process of the original data and the extraction of DTM.

In 2010, Zhou et al. [1] discussed the conformation and flow confluence characteristic of the hill-slope in representative loess landforms and proposed an auto segmentation method of P-N terrains from $5 \mathrm{~m}$ resolution digital elevation models. In 2011, Luo et al. [2] analyzed the advantages and disadvantages of the existing filtering methods and utilized a new adaptive mathematic morphology filtering algorithm. In 2012, Wang et al. [3] combined the mathematical morphology and wavelet domain enhancement and put forward a new algorithm to filter the Gaussian noise in digital images. In 2013, Chen et al. [4] made comparisons of the adaptive triangle irregular network modeling algorithm and threshold segmentation filtering method to deal with the large region and a dramatic topology of LiDAR point cloud. In the same year, Kim et al. [5] proposed a new mean planner filter to generate a digital terrain model and build detection of airborne LiDAR data. Ma and $\mathrm{Li}$ [6] proposed a vegetation filtering method based on the attenuation model of laser light intensity to obtain the high accuracy ground elevation model of the gully area in loess gully region. In 2014, Rau et al. [7] utilized high spatial resolution multispectral optical imagery and a digital elevation model with an object-oriented analysis technique to develop a scheme for the recognition of landslides using multilevel segmentation and a hierarchical semantic network. In 2015 , Su et al. [8] classified non-ground points and ground points and labelled step by step to generate the high-resolution digital models for large areas. In 2016, Zeng et al. [9] presented a filtering method for near-infrared laser scanning data that 
exploits the principle of entropy maximization as the optimization objective. They automatically separated ground and nonground points by the principle of entropy maximization. In the same year, Wang et al. [10] implemented the segmentation of DTM threshold to obtain the real physical model to characterize the pores and fissures of coal mass and to numerically investigate the internal gas flow. Luo et al. [11] proposed the mathematical morphological method to obtain the edges of the continuous, coarser, and smooth features based on the pre-processing and threshold image. In 2017, Hui et al. [12] proposed an improved progressive morphological filter based on hierarchical radial basis function interpolation to generate the digital terrain model from airborne LiDAR data. In the same year, Kim et al. [13] developed a slope-based adaptive principal component analysis-triangular irregular network method to obtain the digital terrain model from the point clouds of steep terrains. Li et al. [14] proposed a novel filter of LiDAR point clouds based on geodesic transformations of mathematical morphology to deal with the dependence on the selection of structuring elements.

The digital terrain model can intuitively reflect the ground information of the earth's surface. Especially in urban planning, the buildings extracted through digital terrain models can be applied to rebuild the 3D model of the city to timely reflect the basic conditions of urban buildings. Since the method of obtaining DTM from laser point cloud data is still immature, it remains a challenging task to separate the surface point and the feature point. Therefore, the TSMM algorithm presented in this paper is of great significance to deal with the extraction of DTM in urban areas.

\section{Basic Theories of the TSMM Algorithm}

\subsection{The Optimal Threshold Segmenting (OTS) Algorithm}

Image segmentation is a process that separates the different characteristics of the region. Threshold segmentation is a method to determine whether the image area is in the target area or the background area through a certain threshold [15]. Therefore, the selection of a proper threshold is the key to the image segmentation, and taking the right way to select the optimal segmenting threshold is the main part of the threshold segmentation. The optimal threshold is the interactive method to select the optimal image segmentation threshold, and it can effectively reduce the noise impact in the image. The steps of the optimal threshold segmentation are shown as follows.

Step 1 Select the highest point and the lowest point in the image, and mark them respectively as $G_{\max }$ and $G_{\min }$.

Step 2 Calculate the current average threshold, and mark it as NowMid $=\left(G_{\max }+G_{\min }\right) / 2$.

Step 3 Calculate the total number (Num_1) of gray values greater than NowMid, and regard HighMid as the geometric average of the region.

Step 4 Calculate the total number (Num_2) of gray values less than NowMid, and regard LowMid as the geometric average of the region.

Step 5 Calculate the area average, and mark it as (HighMid + LowMid $) / 2$.

Step 6 If Num_1 $=0$, Num_2 $=0$, or NowMid and LowMid satisfy the certain accuracy range, the NowMid will be returned. Otherwise, turn to Step 3.

\subsection{The IDP Interpolating Algorithm}

The fundamental of the IDP interpolation algorithm is that the point elevation is calculated through distance weighted interpolation based on the coordinates $(X, Y)$ and the elevations $Z$ of a series of known discrete points distributed in the plane. The expression of the IDP algorithm is shown as Equation (1). It assumes that the attribute value of the point $P_{j}$ is a weighted average of the distances of all the data points in a local neighbourhood. The difference of the distribution position between the surrounding points and the interpolation points has different effects on $Z$, and the effects can be described by the weight function $W$. The interpolated image is smoother and cleaner than before.

$$
G_{i}=\sum_{i=1}^{N} w_{i} \times H_{i}
$$

In Equation (1), $G_{i}$ is the predicted value, $H_{i}$ is the elevation value of the original surrounding point, and $N$ is the number 
of surrounding points. Since the research area is a certain range of rectangles, the expression of weight $w_{i}$ is shown as Equation (2).

$$
w_{i}=\frac{\operatorname{dis}(n) \times d_{i}^{-\theta}}{\sum_{i=1}^{N} \operatorname{dis}(n) \times d_{i}^{-\theta}}
$$

In Equation (2), $d$ is the plane distance between the predicting point and surrounding point and dis $(n)$ is the average distance of sampling points. $w_{i}$ represents the weight, and $\sum_{i=1}^{N} w_{i}=1$. The greater the weight for the point closer to the centre, the lower the weight for the point farther away from the centre. The steps of the IDP interpolation algorithm are shown as follows.

Step 1 Traverse every point in the interpolation range.

Step 2 If the point is a point to be interpolated, traverse all the points around the point and calculate the distance between the center points $d_{i}$.

Step 3 Calculate each weight $w_{i}$ based on Equation (2).

Step 4 Traverse each weight in the interpolation range and calculate the elevation value for the weight based Equation (1).

Step 5 The algorithm ends after traversing the image.

\subsection{Theories of Mathematical Morphology}

Mathematical morphology is a method to filter the elevation image based on the erosion, expansion, and open operations in morphology [16]. Assuming that is a set of images and is a structural element, the erosion operation is to select the minimum value of in the structural element definition field. Its expression is shown as Equation (3), and the calculating process is shown in Table 1.

$$
A \Theta B(s, t)=\min \{A(s+x, t+y)+B(x, y) \mid(s+x, t+y) \epsilon D(A), x, y \epsilon D(B)\}
$$

Table 1. The calculating process of the erosion operation

\begin{tabular}{|l|l|l|l|l|l|l|l|l|}
\hline \multicolumn{4}{|c|}{ Image element (A) } & \multicolumn{3}{c|}{ Structural element (B) } & \multicolumn{3}{c|}{ Result } \\
\hline 29 & 34 & 32 & 1 & 1 & 1 & $?$ & $?$ & $?$ \\
\hline 30 & 31 & 31 & 1 & 1 & 1 & $?$ & 28 & $?$ \\
\hline 28 & 23 & 30 & 1 & 1 & 1 & $?$ & $?$ & $?$ \\
\hline
\end{tabular}

The expansion operation selects the maximum value of $A-B$ in the structural element definition field [17]. Its expression is shown as Equation (4), and the calculating process is shown in Table 2.

$$
A \oplus B(s, t)=\max \{A(s+x, t+y)+B(x, y) \mid(s+x, t+y) \epsilon D(A), x, y \in D(B)\}
$$

Table 2. The calculating process of the expansion operation

\begin{tabular}{|l|l|l|l|l|l|l|l|l|}
\hline \multicolumn{4}{|c|}{ Image element (A) } & \multicolumn{3}{c|}{ Structural element (B) } & \multicolumn{3}{c|}{ Result } \\
\hline 29 & 34 & 32 & 1 & 1 & 1 & $?$ & $?$ & $?$ \\
\hline 30 & 31 & 31 & 1 & 1 & 1 & $?$ & 34 & $?$ \\
\hline 28 & 23 & 30 & 1 & 1 & 1 & $?$ & $?$ & $?$ \\
\hline
\end{tabular}

The expression of open operation in mathematical morphology is shown as Equation (5), and the calculating process is shown as Table 3.

$$
A \circ B=(A \Theta B) \oplus B
$$

According to Table 3, the function of the open operation is to gradually smooth the higher peak and fill the lower points in a continuous three-dimensional image. In this way, the value of the point will always be between the maximum and minimum values in the field. A smooth plane will be formed, and the coordinate of the point in the plane is nearly close to $(x, y, z)$. 
Table 3. The calculating process of the open operation

\begin{tabular}{|c|c|c|c|c|c|c|c|c|c|c|c|c|c|c|}
\hline \multicolumn{5}{|c|}{ Image element (A) } & \multicolumn{5}{|c|}{ Erosion operation } & \multicolumn{5}{|c|}{ Expansion operation } \\
\hline 44 & $\mathrm{X}$ & $\mathrm{x}$ & $\mathrm{x}$ & $\mathrm{x}$ & $\mathrm{y}$ & $\mathrm{y}$ & $\mathrm{y}$ & $\mathrm{y}$ & $\mathrm{y}$ & $\mathrm{Z}$ & $\mathrm{Z}$ & $\mathrm{Z}$ & $\mathrm{Z}$ & $\mathrm{Z}$ \\
\hline $\mathrm{x}$ & 48 & $\mathrm{x}$ & $\mathrm{x}$ & $\mathrm{x}$ & $\mathrm{y}$ & 44 & $\mathrm{y}$ & $\mathrm{y}$ & $\mathrm{y}$ & $\mathrm{Z}$ & $\mathrm{Z}$ & $\mathrm{Z}$ & $\mathrm{Z}$ & $\mathrm{Z}$ \\
\hline $\mathrm{x}$ & $\mathrm{x}$ & 50 & $\mathrm{x}$ & $\mathrm{x}$ & $\mathrm{y}$ & $\mathrm{y}$ & 40 & $\mathrm{y}$ & $\mathrm{y}$ & $\mathrm{z}$ & $\mathrm{Z}$ & 44 & $\mathrm{z}$ & $\mathrm{z}$ \\
\hline $\mathrm{x}$ & $\mathrm{x}$ & $\mathrm{x}$ & 40 & $\mathrm{x}$ & $\mathrm{y}$ & $\mathrm{y}$ & $\mathrm{y}$ & 28 & $\mathrm{y}$ & $\mathrm{z}$ & $\mathrm{Z}$ & $\mathrm{Z}$ & $\mathrm{z}$ & $\mathrm{z}$ \\
\hline $\mathrm{x}$ & $\mathrm{x}$ & $\mathrm{x}$ & $\mathrm{x}$ & 28 & $\mathrm{y}$ & $\mathrm{y}$ & $\mathrm{y}$ & $\mathrm{y}$ & $\mathrm{y}$ & $\mathrm{z}$ & $\mathrm{Z}$ & $\mathrm{Z}$ & $\mathrm{z}$ & $\mathrm{z}$ \\
\hline
\end{tabular}

\section{Design of DTM Extraction Algorithm and 3D Visualization}

\subsection{Design of the OTS-EO Algorithm}

In this paper, the optimal segmenting threshold is obtained through multiple scene segmentation and regarded as the rejection threshold. After that, the threshold is optimized by the erosion algorithm to obtain the filtered image. The idea of optimal segmenting threshold is described as follows.

Find the current optimal segmenting threshold to determine whether the point threshold is greater than the current optimal segmenting threshold and meets the accuracy requirements. If the point threshold is greater, the current optimal segmenting threshold will be regarded as the maximum or the minimum of the threshold to continue the segmentation. The segmentation will be ended when the current optimal segmenting threshold is satisfied with the accuracy requirements.

If the threshold of a certain point is higher than the optimal threshold calculated by the above method, it will be taken into an erosion operation as Equation (6).

$$
g\left(f, k_{0}, \lambda, b\right)=\left\{\begin{array}{r}
f, f<T \\
f \Theta b, f \geq T
\end{array}\right.
$$

In Equation (6), $\mathrm{g}$ represents the image operated by erosion operation and $\mathrm{T}$ is the optimal segmenting threshold obtained by the above method. $\mathrm{b}$ represents the structural element, and $\mathrm{f}$ is the elevation of the original image. Then, the optimal threshold of the image will be found to remove the points that meet the needs, until no points need to be removed.

The flowchart of the optimal threshold segmenting filter algorithm based on erosion operation is shown in Figure 1.

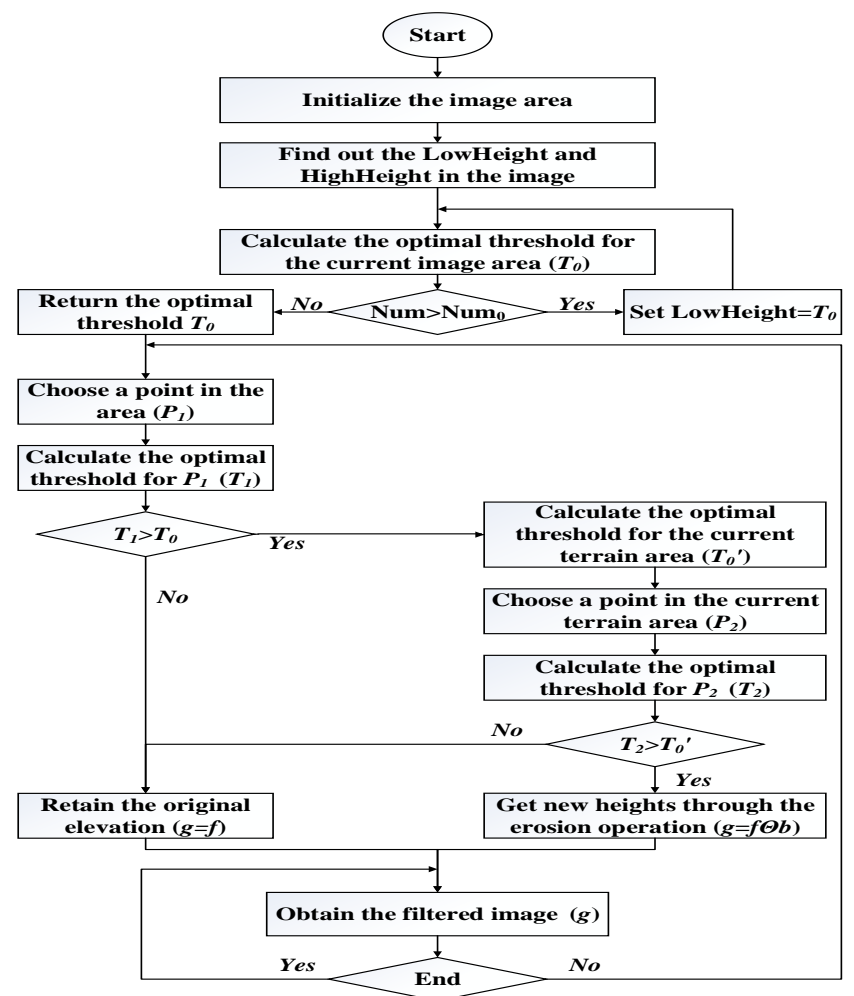

Figure 1. The flowchart of the optimal threshold segmenting filter algorithm based on erosion operation 


\subsection{Design of the Interpolation Algorithm based on Elevation and Distance Weighting}

The IDP algorithm is an interpolation algorithm based on the distance and the surrounding information. In this algorithm, the closer the point to the center, the higher the weight will be. However, the IDP interpolation algorithm only refers to the distance between surrounding points and the center of the plane, and it ignores the impact of elevation information on the interpolation point. Therefore, this paper proposes the interpolation algorithm based on the elevation and distance weighting, and the idea of the algorithm is described as follows.

First, the maximum coordinates and the minimum coordinates of the scanning area are found and marked as $X_{\min }, Y_{\min }, Z_{\min }, X_{\max }, Y_{\max }, Z_{\max }$. Then, the whole point cloud data is divided into grids of a certain size. Finally, the weight of the information of elevation and distance is set, and the expression is shown as Equation (7). The expression of elevation information is shown as Equation (8).

$$
\begin{gathered}
W=\sum_{k=1}^{n_{i j}} W_{i j k} \\
G_{i j}=\sum_{k=1}^{n_{i j}} W_{i j k} Z_{i j k} / W
\end{gathered}
$$

In the Equation (7), $n_{i j}$ represents the point involved in the operation and $W_{i j k}$ is the weight of the point. In Equation (8), $Z_{i j k}$ is the elevation value of the point and $G_{i j}$ represents the estimating value. The expression of $W_{i j k}$ is shown as Equation (9).

$$
W_{i j k}=\alpha W_{i j k}^{x y}+\beta W_{i j k}^{h}
$$

In Equation (9), $\alpha$ is the weight coefficient of the plane and $\beta$ is the weight coefficient of the elevation. $\alpha$ and $\beta$ follow the principle that $\alpha+\beta=1$, and the value of $\alpha$ is 0.75 in this paper.

$$
\begin{gathered}
W_{i j k}^{x y}=\sqrt{2} G S D / D_{i j}^{k} \\
W_{i j k}^{h}=\frac{\left(Z_{i j k}-h_{\min }\right)\left(h_{\min (i j)}-Z_{\min }\right)}{\left(Z_{\max }-h_{\max (i j)}\right)}
\end{gathered}
$$

In Equation (10), GSD represents the grid interval and $Z_{i j k}$ is the $k^{\text {th }}$ point in the grid $(i, j)$. In Equation $(11), h_{\min (i j)}$ is the lowest elevation in the grids and $h_{\max (i j)}$ is the highest elevation in the grids. Besides, $Z_{\min }$ is the lowest elevation of the area and $Z_{\max }$ is the highest elevation of the area.

As a result, the calculation of the eigenvalue of the interpolating point is divided into two parts. The first part of the calculation is determined by the distance $D_{i j}^{k}$ between the point in the grid and the center of the plane $X O Y$. The second part of the calculation is determined by the difference of elevation $h_{i j}^{k}$ between the point in the grid and the lowest point in the grid. The flowchart of the interpolation algorithm based on elevation and distance weighting is shown in Figure 2.

\subsection{Design of the DTM Extracting Algorithm based on TSMM}

In this paper, a small window is set for initial filtering, and the threshold of the optimal segmenting point is used for the threshold segmentation. The threshold $M$ of the segmenting point is obtained by referring to the current situation and constantly iterating. An upper limit parameter is set based on the optimal segmenting threshold to take the laser points that meet the condition $G(x, y)>M \times \Psi$ into open operation. The point that cannot meet the condition remains the same. In this way, it can be ensured that the smaller terrain will not be removed, and the larger area can be filtered. In order to ensure the adaptability of the DTM, this paper makes use of the rectangular structure elements, and the expression is shown as Equation (12). 


$$
g(f, M, \Psi, b)=\left\{\begin{array}{r}
f, k<M \times \Psi \\
f \circ b, k \geq M \times \Psi
\end{array}\right.
$$

In Equation (12), $M$ represents the optimal segmenting threshold and $\Psi$ is the upper limit parameter. The optimal threshold segmentation and filtering method does not need to divide the image area several times, and the flowchart of the TSMM algorithm is shown in Figure 3.

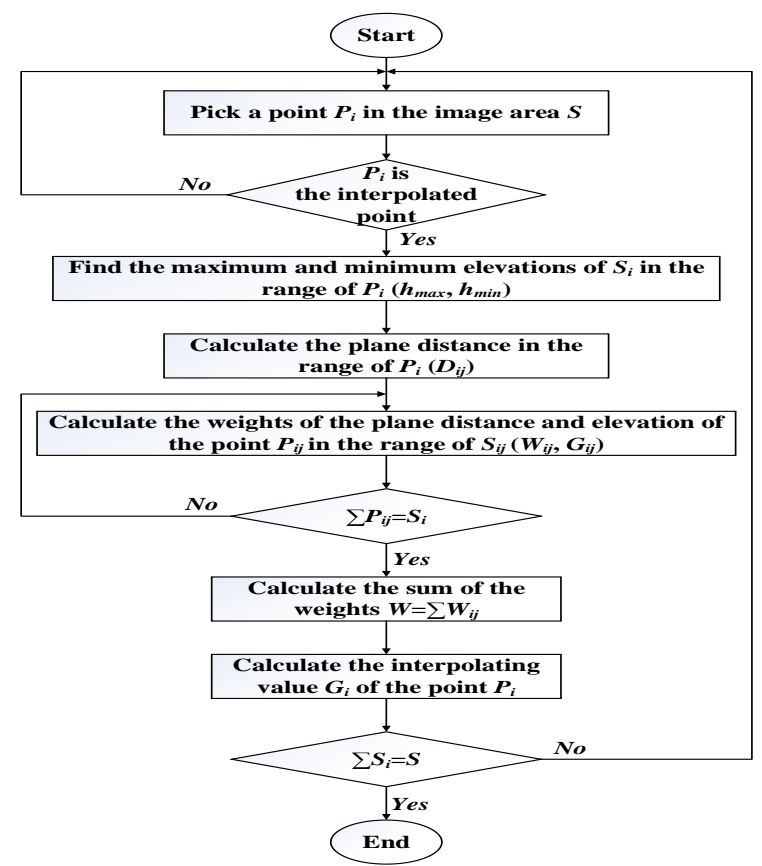

Figure 2. The flowchart of the interpolating algorithm based on elevation and distance weighting

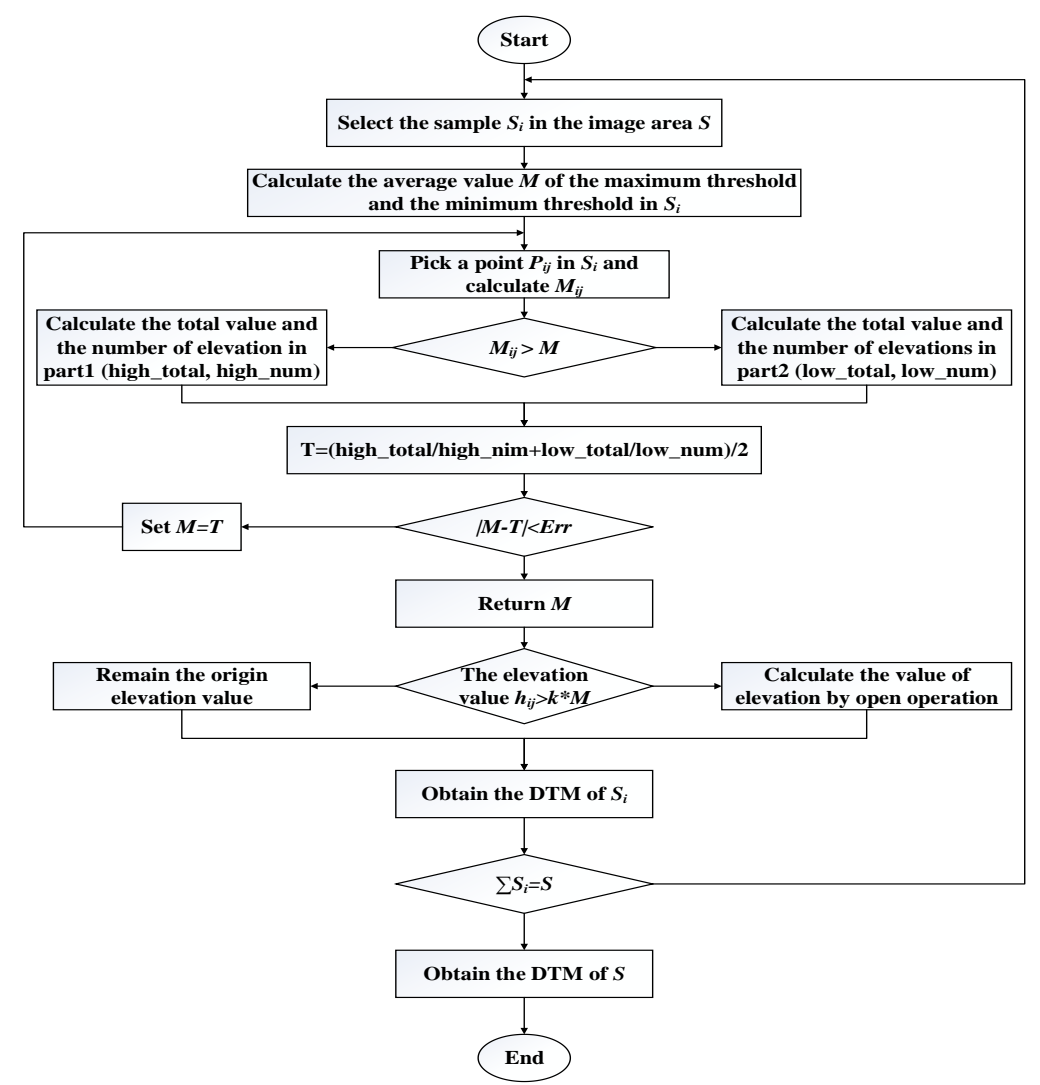

Figure 3. The flowchart of the TSMM algorithm 


\section{Experimental Results and Comparison Analysis of the Case}

\subsection{Comparison and Analysis of the Case}

The LiDAR ACSSI data read in this paper is after the process of coordinate transformation and Gaussian projection, and the program based on MFC and OpenGL is developed to realize the 3D visualization of terrain. The two-dimensional map of the test data is shown in Figure 4, and the three-dimensional map of the test data is shown in Figure 5.

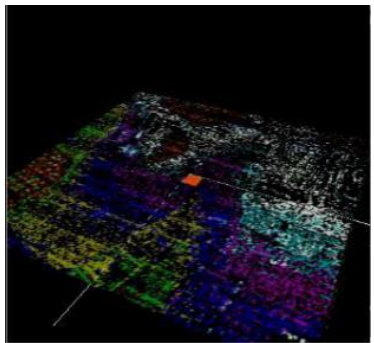

Figure 4. The two-dimensional map of the test data

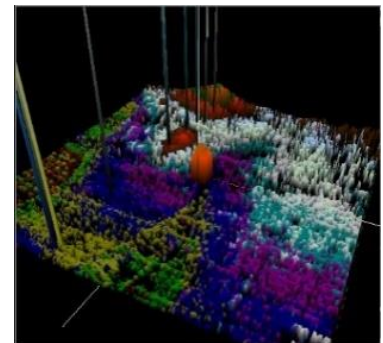

Figure 5. The three-dimensional map of the test data

According to Figures 4 and 5, there are many missing cases in the two-dimensional map, and there is a number of test data in an abnormal situation in the three-dimensional map.

\subsubsection{Comparison of Filter Processing Results}

In this paper, the three-dimensional map of the test data is filtered through the optimal threshold segmenting algorithm, and the results of the elimination for the abnormal points are shown in Figure 6. Then, the optimal threshold segmenting algorithm based on the erosion operation is applied to filter the three-dimensional map of the test data, and the results of the filter operation are shown in Figure 7.

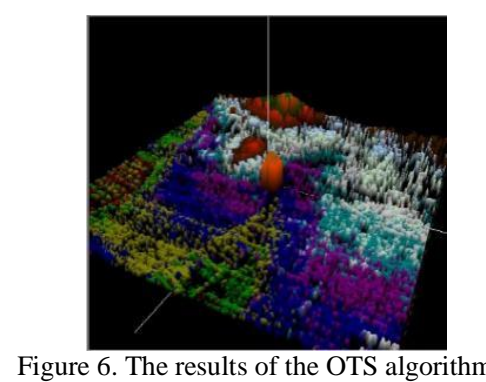

According to Figure 6 and

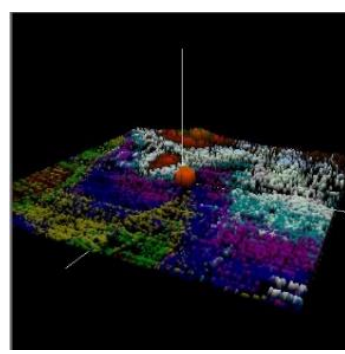

Figure 7. The results of the OTS-EO algorithm

abnormal points, but the OTS-EO algorithm has obvious advantages in accuracy and preventability of the misjudgement of the terrain with higher points and certain slopes. In order to intuitively reflect the advantages of the OTS-EO algorithm, a partial comparison is made to discuss the effect before and after the filtering processing, and the results are shown as Figure 8 and Figure 9.

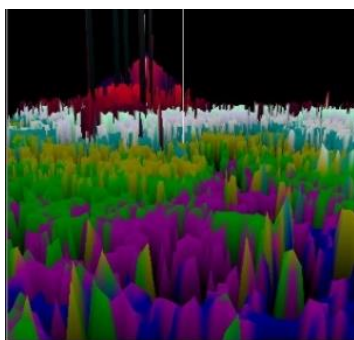

Figure 8 . The results before the filtering processing

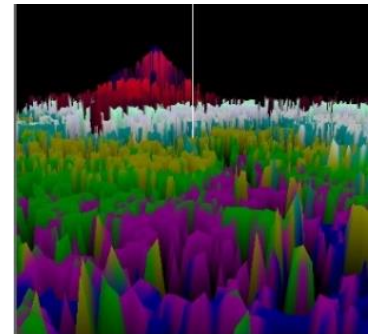

Figure 9. The results after the filtering processing

According to Figure 8 and Figure 9, the OTS-EO algorithm can deal with the global redundant point and maintain the steep gradient at the same time. 


\subsubsection{Comparison of Interpolation Results}

In this paper, the IDP interpolating algorithm is applied to interpolate the two-dimensional map of the test data, and the interpolating result is shown in Figure 10. Then, the interpolating algorithm based on elevation and distance weighting is also utilized for the interpolation of the two-dimensional map of the test data, and the interpolating result is shown in Figure 11.

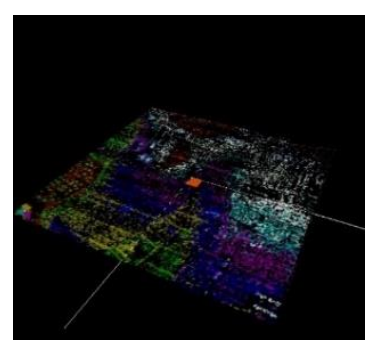

Figure 10. The results of IDP interpolating algorithm

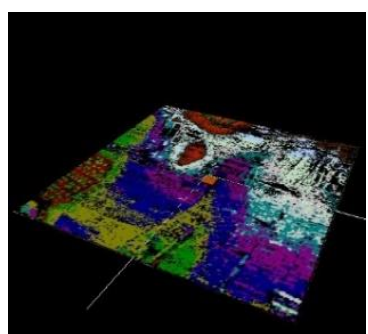

Figure 11. The results of the interpolating algorithm based on elevation and distance weighting

According to Figure 10 and Figure 11, although the IDP interpolating algorithm can fill some missing data, the interpolating effect is not ideal for the discrete original image. The interpolating algorithm based on elevation and distance weighting can better deal with the case of discrete distribution, and it can interpolate without losing the accuracy of the image.

\subsubsection{Comparison of DTM Extraction Results}

In order to compare the DTM extracting ability of the two algorithms, partial feature samples are selected from the threedimensional image to conduct the process of filtering and interpolating. The color table and the texture are shown in Figure 12.

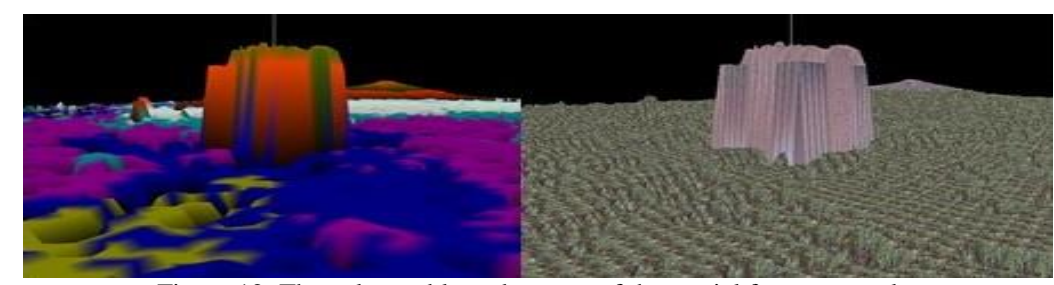

Figure 12. The colour table and texture of the partial feature samples

When the OTS algorithm is utilized to extract the DTM, the colour table and the texture after extraction are shown in Figure 13.

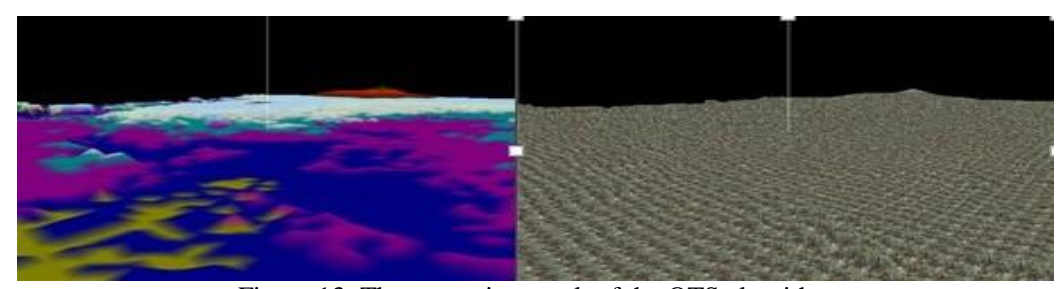

Figure 13. The extracting result of the OTS algorithm

When the TSMM algorithm is applied to extract the DTM, the colour table and the texture after one iteration, two iterations, and four iterations are shown in Figure 14 to Figure 16.

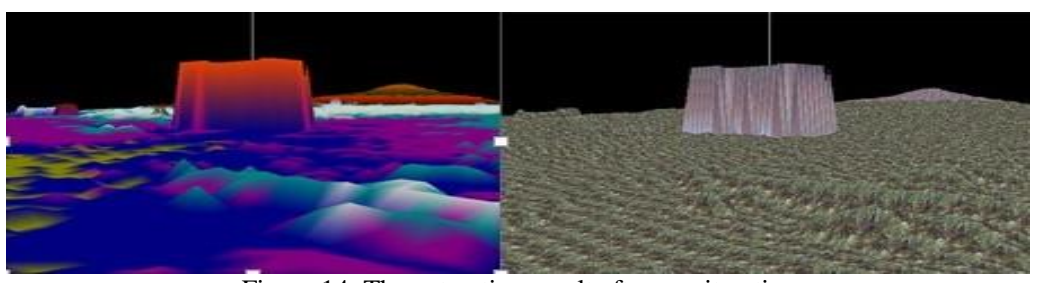

Figure 14. The extracting result after one iteration 


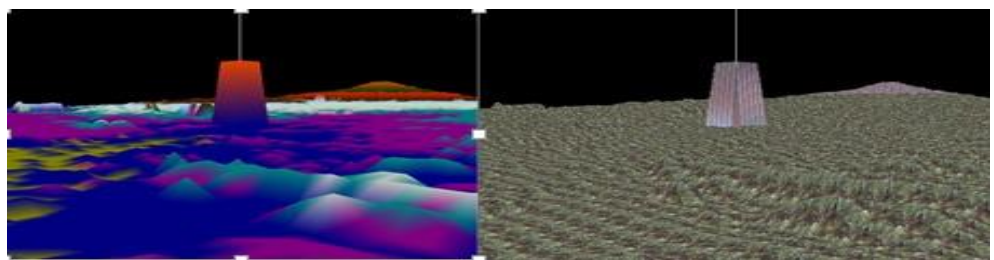

Figure 15. The extracting result after two iterations

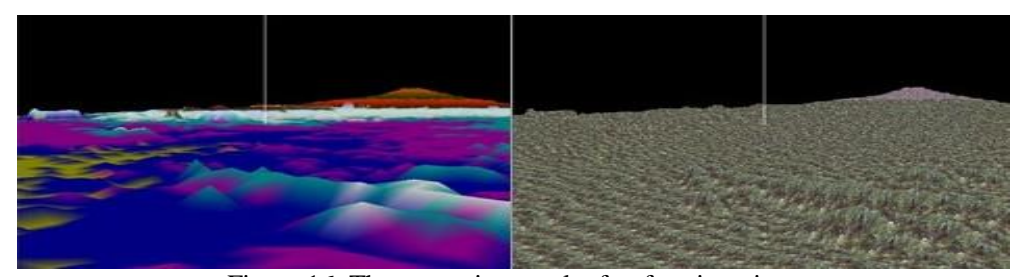

Figure 16. The extracting result after four iterations

According to Figure 13, the OTS algorithm can effectively filter out the feature points and reserve some low reliefs, but some of the higher terrain are removed. It can be seen from Figure 14 to 16 that the TSMM algorithm can not only preserve the low relief, but also avoid the higher terrain being misjudged as a feature point. In this way, the accuracy of DTM extraction can be effectively improved.

\subsection{Engineering Actual Test of the TSMM Algorithm}

In order to test the practicality of the TSMM algorithm, the original laser point cloud data near Xinyan Road in Beijing is selected as an example to finally verify whether the features are effectively eliminated. The original laser point cloud data is shown in Figure 17, and the DSM generated by the original point cloud data is shown in Figure 18.

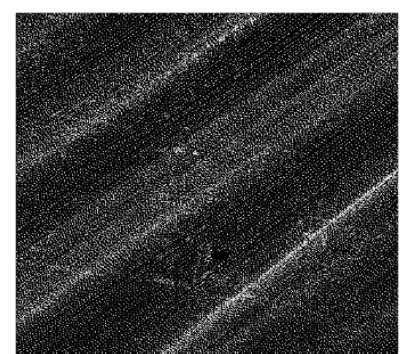

Figure 17. The original laser point cloud data

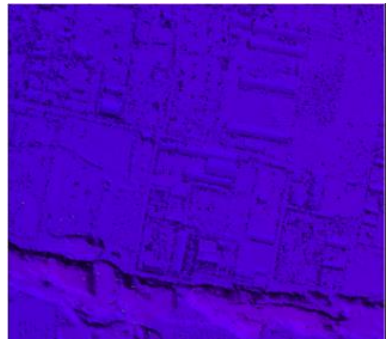

Figure 18. The DSM generated by the original laser point cloud data

After the process of five iterations and ten iterations based on the TSMM algorithm, the results of the DSM generated by the original laser point cloud data are shown in Figure 19 and Figure 20. The final results after twenty iterations based on the TSMM algorithm are shown in Figure 21.

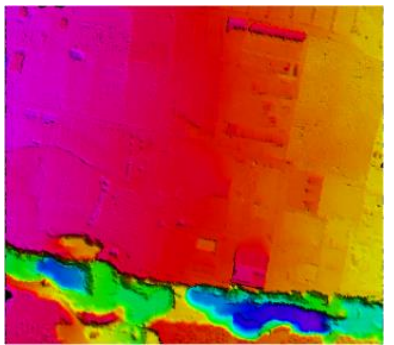

Figure 19. The result of DSM after five iterations

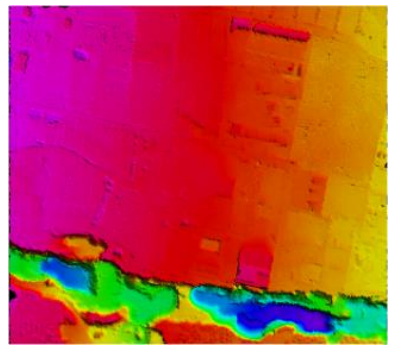

Figure 20. The result of DSM after ten iterations

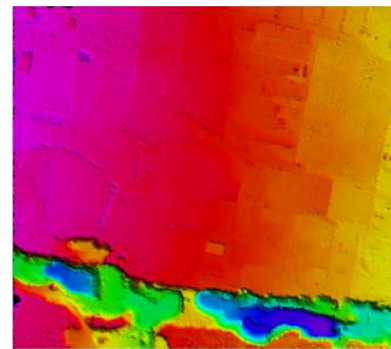

Figure 21. The final result of original DSM after twenty iterations

Taking Figure 18 and Figure 21 into comparison, the buildings of the original data are effectively filtered out and the terrain is still retained. Therefore, the TSMM algorithm can effectively eliminate the feature points and preserve the undulating information of the higher slope of the ground, and it is a good method for the extraction of the digital terrain model. 


\section{Conclusions}

This paper first proposed the OTS-EO filtering algorithm to overcome the shortcomings of the optimal threshold segmenting algorithm, and the original laser point cloud data was filtered. The interpolating algorithm based on height and distance weighting was presented to improve the problem that the IDP algorithm cannot effectively interpolate discrete point cloud data. The TSMM extracting algorithm was then put forward to extract the DTM from the original DSM after the process of filtering and interpolating. Finally, the 3D visualization of digital terrain model was realized through the program based on MFC and OpenGL. The experimental results showed that the TSMM algorithm proposed in this paper can effectively remove the feature points and preserve the topographic undulation information of the higher gradient of the ground surface.

\section{Acknowledgements}

The authors are grateful for the area editor and the anonymous reviewers for their valuable comments and suggestions. They would also like to show their appreciation for the original data and professional help provided by China Communications Section (Beijing) Space Information Technology Co., Ltd.

\section{References}

1. Y. Zhou, G. A. Tang, C. Wang, C. C. Xiao, Y. F. Dong, and J. L. Sun, "Automatic Segmentation of Loess Positive and Negative Terrains based on High Resolution Grid DEMs," Scientia Geographica Sinica, Vol. 30, No. 2, pp. 261-266, 2010

2. Y. P. Luo, T. Jiang, X. Wang, R. Zhang, and S. Luo, "A New Filtering Method for LiDAR Data based on Mathematic Morphological Approach," Bulletin of Surveying and Mapping, No. 3, pp. 15-19, 2011

3. X. B. Wang, J. Y. Sun, and H. Y. Tang, "A New Filter Algorithm based on Mathematical Morphology and Wavelet Domain Enhancement," Microelectronics \& Computer, Vol. 29, No. 7, pp. 64-67, 2012

4. L. Chen, X. T. Fan, and X. P. Du, "Comparisons of Adaptive TIN Modelling Filtering Method and Threshold Segmentation Filtering Method of LiDAR Point Cloud," in Proceedings of the 8th International Symposium of the Digital Earth, 2013

5. Y. M. Kim, Y. D. Eo, A. J. Chang, and Y. I. Kim, "Generation of A DTM and Building Detection based on An MPF through Integrating Airborne LiDAR Data and Aerial Images,” International Journal of Remote Sensing, Vol. 34, No. 8, pp. 2947-2968, 2013

6. D. Ma and B. B. Li, "Vegetation Filtering in Gully Region of Loess Plateau based on Laser Scanning Point Cloud's Intensity Attenuation Model and its Terrain Construct," Transactions of the Chinese Society of Agricultural Engineering, Vol. 29, No. 15, pp. 162-171, 2013

7. J. Y. Rau, J. P. Jhan, and R. J. Rau, "Semiautomatic Object-Oriented Landslide Recognition Scheme from Multisensor Optical Imagery and DEM," IEEE Transactions on Geoscience and Remote Sensing, Vol. 52, No. 2, pp. 1336-1349, 2014

8. W. Su, Z. P. Sun, R. F. Zhong, J. X. Huang, and M. L. Li, “A New Hierarchical Moving Curve-Fitting Algorithm for Filtering LiDAR Data for Automatic DTM Generation,” International Journal of Remote Sensing, Vol. 36, No. 14, pp. 3616-3635, 2015

9. Z. Zeng, J. X. Wan, and H Liu, "An Entropy-based Filtering Approach for Airborne Laser Scanning Data," Infrared Physics \& Technology, Vol. 75, pp. 87-92, 2016

10. G. Wang, X. X. Yang, X. Q. Zhang, W. X. Li, and L. K. Shi, "Numerical Simulation of Gas Flow in Pores and Fissures of Coal based on Segmentation of DTM Threshold," Chinese Journal of Rock Mechanics and Engineering, Vol. 35, No. 1, pp. 119-129, 2016

11. J. H. Luo, J. P. Jiang, and P. M. Zhu, "Automatic Extraction of The Side-Scan Sonar Imagery Outlines based on Mathematical Morphology," Haiyang Xuebao, Vol. 38, No. 5, pp. 150-157, 2016

12. Z. Y. Hui, B. P. Wu, Y. J. Hu, and Y. Y. Ziggah, "Improved Progressive Morphological Filter for Digital Terrain Model Generation from Airborne LiDAR Data," Applied Optics, Vol. 56, No. 34, pp. 9359-9367, 2017

13. M. K. Kim, S. Kim, H. G. Sohn, N. Kim, and J. S. Park, "A New Recursive Filtering Method of Terrestrial Laser Scanning Data to Preserve Ground Surface Information in Steep-Slope Areas," ISPRS International Journal of Geo-Information, Vol. 6, No. 11, pp. 359, 2017

14. Y. Li, B. Yong, O. P. Van, and M. Lemmens, "Airborne LiDAR Data Filtering based on Geodesic Transformations of Mathematical Morphology," Remote Sensing, Vol. 9, No. 11, pp. 1104, 2017

15. S. H. Yang, "Study on the Adaptive and Fast Algorithm of Gray Scale Image Thresholding," Chongqing University, 2014

16. M. B. Zhao, J. He, J. S. Tian, and Q. Fu, "Ladar Data Filtering Method based on Improved Progressive Multi-Scale Mathematic Morphology," Acta Optica Sinica, Vol. 33, No. 3, pp. 285-294, 2013

17. Q. Fang, S. S Zhang, and C. Duan, "Dilation and Erosion Operation based Bridge Detection in SAR Image," Fire Control Radar Technology, Vol. 40, No. 3, pp. 37-41, 2011

Tianyong Wu is a faculty member at the China University of Geosciences. His research interests include machine learning and neural network algorithms.

Yunsheng Zhao graduated from the China University of Geosciences with a bachelor's degree, Master's degree, and Ph.D. 
He is currently a professor at the China University of Geosciences and the director of the Department of Safety Engineering. His current research interests include system security theory and applications, safety information engineering, and occupational safety and health.

Xiang Li is a professor in the School of Computer Science at the China University of Geosciences. His research interests include neural network algorithms, remote sensing classification, and recognition. 